\title{
Could the Ultrasound Interscalene Block continuous be a strategy for pain management and reduce the complications in critical care patient with acute renal failure?
}

C. Giordano $^{1}$, E. Centonze ${ }^{2}$, A. Liberto ${ }^{1}$, P. Scimia 1 , V. Danzi ${ }^{1}$.

${ }^{1}$ Hospital of Cremona, Anesthesia and Intensive Care Unit, Cremona, Italy.

${ }^{2}$ Hospital University of Parma, Anestesia and Intensive Care Unit, Parma, Italy.

\section{Background and Aims:}

Regional analgesia with continuous dosing using peripheral catheters can play an important role in pain management and can reduce the complications in critical care patient.

We performed Ultrasound Interscalene Block with continuous catheter in patient with acute respiratory and renal failure admitted to the Intensive Care Unit (ICU) after shoulder surgery for fracture traumatic.

\section{Methods:}

A 85 years old, female, ASA 3, with chronic renal failure, scheduled to undergo right shoulder surgery because of accidental fall.After surgery the patient presented acute renal and respiratory failure and was transferred to ICU.The patient was intubated and connected to ventilator machine and continuous venovenous haemodiafiltration (CVVHDF) was initiated.

During the recovery, continuous infusion of morphine to $0,8 \mathrm{mg} / \mathrm{h}$ was administered for analgesia.We decided to stop infusion of morphine and we performed US-interscalene block continuous with electrical neurostimulation for avoid the block of the ipsilateral phrenic nerve, which could determine difficult weaning from mechanical ventilation.

\section{Results:}

After two days the patient was extubated, presented good pain control and CVVHDF was interrupted with restore normal renale function.

\section{Conclusions:}

This case report could demonstrated that regional analgesia techniques provide numerous clinical benefits to critical care patients such as improvement in pain management and less pulmonary and renale complications.

Keywords:

Ultrasound Interscalene Block continuous,critical care patient,acute renal failure. 